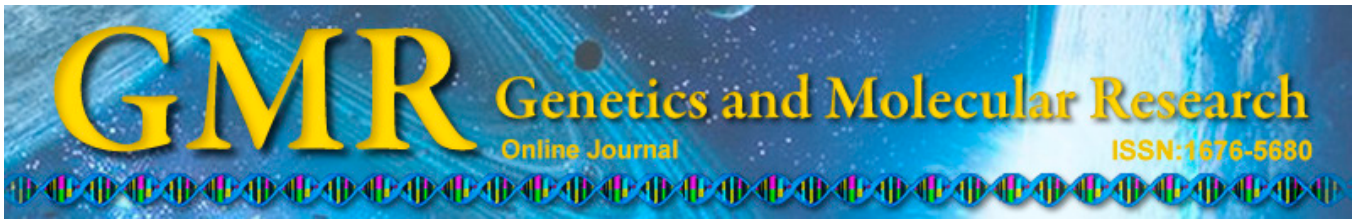

\title{
Association between MTHFR 677C/T polymorphism and psoriasis risk: a meta-analysis
}

\author{
J.H. Qi ${ }^{1}$, J.H. Qi ${ }^{2}$, N. Shi ${ }^{3}$, Y.J. Chen ${ }^{3}$ and G. Nie \\ ${ }^{1}$ Department of Hubei Key Laboratory of Kidney Disease Pathogenesis and \\ Intervention, Huangshi Central Hospital, \\ Affiliated Hospital of Hubei Polytechnic University, Huangshi, China \\ ${ }^{2}$ Department of Burns, People's Hospital of Lanling County, Lanling, China \\ ${ }^{3}$ Department of Dermatology, Huangshi Central Hospital, \\ Affiliated Hospital of Hubei Polytechnic University, Huangshi, China \\ Corresponding author: G. Nie \\ E-mail: niegang2014@126.com
}

Genet. Mol. Res. 14 (2): 3869-3876 (2015)

Received August 12, 2014

Accepted December 8, 2014

Published April 22, 2015

DOI http://dx.doi.org/10.4238/2015.April.22.16

\begin{abstract}
Previous studies investigating the association between methylenetetrahydrofolate reductase (MTHFR) $677 \mathrm{C} / \mathrm{T}$ polymorphisms and psoriasis risk have reported inconsistent results. The present metaanalysis aimed to comprehensively evaluate the association between MTHFR $677 \mathrm{C} / \mathrm{T}$ polymorphism and psoriasis risk. The studies regarding the association between $M T H F R$ 677C/T polymorphism and psoriasis risk were retrieved from the PubMed, Embase, Web of Science, Chinese National Knowledge Infrastructure, and Chinese Biomedical databases. Data were extracted and statistical analysis was performed with the program STATA 12.0. A total of seven studies involving 1290 psoriasis cases and 1068 healthy controls were retrieved. Combined analysis showed that there was no significant difference in MTHFR $677 \mathrm{C} / \mathrm{T}$ genotype distribution between psoriasis and control subjects in the comparisons $\mathrm{C}$ vs T, CC vs $\mathrm{CT}+\mathrm{TT}, \mathrm{CC}+\mathrm{CT}$ vs TT, CC vs TT,
\end{abstract}


and $\mathrm{CC}$ vs $\mathrm{CT}$ [respectively: odds ratio $(\mathrm{OR})=0.98,95 \%$ confidence interval $(\mathrm{CI})=0.76-1.26, \mathrm{P}=0.882 ; \mathrm{OR}=1.11,95 \% \mathrm{CI}=0.81-1.51, \mathrm{P}$ $=0.526 ; \mathrm{OR}=0.79,95 \% \mathrm{CI}=0.53-1.19, \mathrm{P}=0.261 ; \mathrm{OR}=0.88,95 \% \mathrm{CI}$ $=0.51-1.52, \mathrm{P}=0.648 ; \mathrm{OR}=1.19,95 \% \mathrm{CI}=0.90-1.58, \mathrm{P}=0.217]$. Subgroup analysis by ethnicity also showed no significant association between MTHFR $677 \mathrm{C} / \mathrm{T}$ polymorphism and psoriasis risk in both Asian and Caucasian populations. In conclusion, this meta-analysis indicates that MTHFR $677 \mathrm{C} / \mathrm{T}$ polymorphism may not be associated with psoriasis risk.

Key words: Methylenetetrahydrofolate reductase; Polymorphism; Psoriasis; Meta-analysis

\section{INTRODUCTION}

Psoriasis is a common chronic and recurrent inflammatory skin disease characterized by epidermal hyperplasia, abnormal keratinocyte differentiation, and local accumulation of acute and chronic inflammatory cells (Krueger et al., 1990; Zenz et al., 2005), which affects approximately $2 \%$ of the population worldwide (Ni and Chiu, 2014). Although the exact pathogenesis of the disease has not yet been clarified, it is widely accepted that genetic, environmental, and immunological factors act together or individually to precipitate the disease (Krueger and Bowcock, 2005).

Methylenetetrahydrofolate reductase (MTHFR), a crucial enzyme in the metabolism of folate, catalyzes the conversion of 5,10-methylenetetrahydrofolate to 5-methyltetrahydrofolate, which is the precursor of S-adenosylmethionine (SAM). SAM is a universal methyl donor for methylation reactions, including DNA methylation (Selhub and Miller, 1992). The MTHFR gene is located at chromosome 1 (1p36.3). The most studied C677T polymorphism in the MTHFR gene results in a thermolabile variant (T) with reduced activity of the enzyme (Friso et al., 2002).

A significant association between MTHFR 677C/T gene polymorphism and psoriasis was reported in a Chinese population (Baiqiu et al., 2000). This finding seemed to be biologically plausible, as MTHFR is associated with DNA methylation reactions (Stern et al., 2000) and DNA methylation disorders might play a role in the etiopathogenesis of psoriasis (Ruchusatsawat et al., 2006; Zhang et al., 2007). Moreover, a lower frequency of the tyrosine phosphatase $(S H P-1)$ gene and 16 gene demethylation has been observed in psoriatic skin lesions (Ruchusatsawat et al., 2006; Zhang et al., 2007). However, several case-control studies carried out subsequently have reported inconsistent results (Weger et al., 2008; Vasku et al., 2009; Liew et al., 2012; Asefi et al., 2014). Therefore, we performed the present meta-analysis to comprehensively evaluate the association between the MTHFR C677T polymorphism and psoriasis risk based on all eligible case-control studies.

\section{MATERIAL AND METHODS}

\section{Literature search}

The studies regarding the association between MTHFR gene 677C/T polymorphism and psoriasis risk published up to May 2014 without language restrictions were independently 
searched by two authors in the PubMed, Embase, Web of Science, Chinese National Knowledge Infrastructure (CNKI), and Chinese Biomedical (CBM) Literature Database, using the following terms: ("MTHFR" or "methylenetetrahydrofolate reductase") and ("psoriasis" or "psoriasis" or "psoriatic") and ("polymorphism" or "SNP" or "single nucleotide polymorphism" or "variation" or "mutation"). The bibliographies of retrieved articles were manually searched to find additional relevant studies.

\section{Study selection}

Studies were included in this meta-analysis if they met the following criteria: (a) casecontrol studies focused on associations between MTHFR $677 \mathrm{C} / \mathrm{T}$ polymorphism and psoriasis risk; (b) 95\% confidence intervals (CIs) for odds ratios (ORs) were available or could be calculated; (c) the distribution of genotypes in the control group was consistent with the HardyWeinberg equilibrium (HWE). When we retrieved repetitive publications, only one publication was included. Family-based studies were excluded.

\section{Data extraction}

The following data from the included studies were extracted independently by two authors: the first author, the year of publication, the country of subject recruitment, the subjects' ethnicities, the sample size, and the genotype frequencies in psoriasis cases and controls. In cases of conflicting evaluation, disagreements were resolved through discussion between the authors.

\section{Statistical analysis}

Genotype distributions in the controls were tested for HWE using the Pearson $\chi^{2}$ test (Schaid and Jacobsen, 1999). Between-study heterogeneity was checked using the Cochran Q-statistic and the $I^{2}$ test (Higgins and Thompson, 2002; Zintzaras and Ioannidis, 2005). When $\mathrm{P}<0.1$ for the Q-test or $I^{2}>50 \%$ indicated the existence of heterogeneity, a randomeffects model was used; otherwise, a fixed-effects model was applied. ORs with corresponding $95 \% \mathrm{CIs}$ were calculated to assess the association between MTHFR gene $677 \mathrm{C} / \mathrm{T}$ polymorphism and psoriasis risk in five genetic models: $\mathrm{C} v s \mathrm{~T}, \mathrm{CC} v s \mathrm{CT}+\mathrm{TT}, \mathrm{CC}+\mathrm{CT} v s \mathrm{TT}, \mathrm{CC} v s$ TT and CC vs CT. The significance of the pooled ORs was determined using the Z-test. To evaluate whether the association showed any ethnicity-specific effects, we analyzed the data for separate subgroups defined by ethnicity. Sensitivity analysis was performed by sequential omission of individual studies and recalculating the results to assess the stability of the results. Begg's funnel plots and the Egger tests were used to investigate whether publication bias might affect the validity of the estimates (Peters et al., 2006). All the statistical tests were conducted using the STATA 12.0 software.

\section{RESULTS}

\section{Characteristics of studies included}

The flow chart of the selection of studies and specific reasons for exclusion from the meta-analysis are shown in Figure 1. 


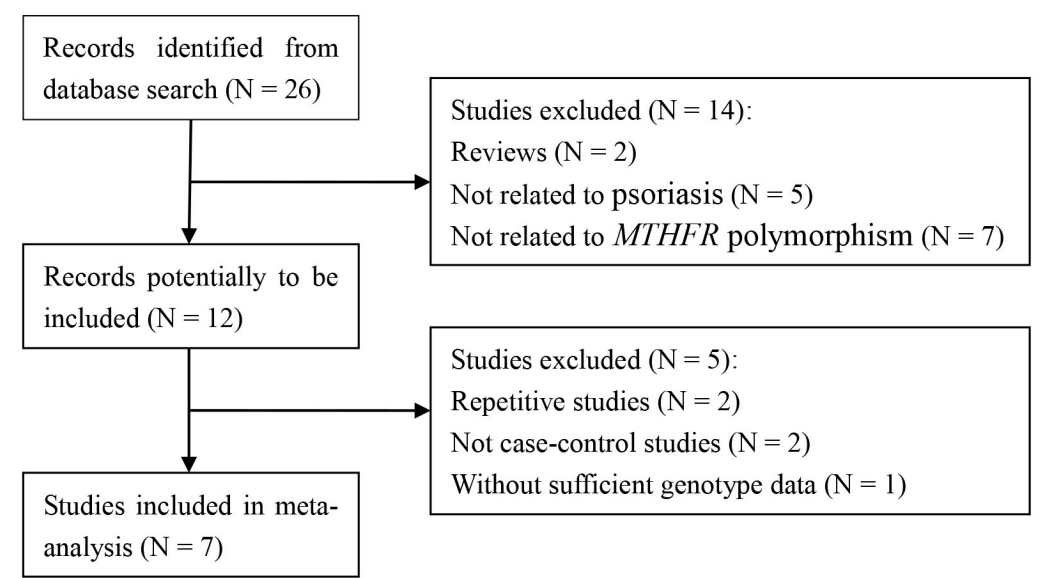

Figure 1. Flow chart of the selection of studies and specific reasons for exclusion from the meta-analysis.

The search strategy retrieved 26 potentially relevant studies. In accordance with the inclusion criteria, seven case-control studies with 1290 psoriasis cases and 1068 healthy controls were included in this meta-analysis. Of the seven eligible studies, five were conducted in Asian populations and the remaining two were conducted in Caucasian populations. The publication year of the included studies ranged from 2000 to 2014. Five articles were written in English and the remaining two were written in Chinese. The distribution of genotypes in the control group of each included study was consistent with HWE (all P $>0.05$ ). The control group was chosen from healthy individuals without any systemic or dermatologic diseases. The characteristics of the studies included are summarized in Tables 1 and 2.

Table 1. Characteristics of the seven studies included in this meta-analysis.

\begin{tabular}{|c|c|c|c|c|c|c|}
\hline First Author (year) & Country & Ethnicity & Source of controls & Sample & Cases & Controls \\
\hline Baiqiu et al. (2000) & China & Asian & Population-based & Blood & 39 & 79 \\
\hline Jie et al. (2007) & China & Asian & Population-based & Blood & 123 & 129 \\
\hline Weger et al. (2008) & Austria & Caucasian & Population-based & Blood & 310 & 247 \\
\hline Vasku et al. (2009) & Czech & Caucasian & Population-based & Blood & 410 & 244 \\
\hline Dehui et al. (2012) & China & Asian & Population-based & Blood & 108 & 102 \\
\hline Liew et al. (2012) & Malaysia & Asian & Population-based & Blood & 200 & 167 \\
\hline Asefi et al. (2014) & Iran & Asian & Population-based & Blood & 100 & 100 \\
\hline
\end{tabular}

Table 2. Distribution of MTHFR $677 \mathrm{C} / \mathrm{T}$ polymorphism in psoriasis patients and control subjects.

\begin{tabular}{|c|c|c|c|c|c|c|c|c|c|c|c|}
\hline \multirow[t]{3}{*}{ Study } & \multicolumn{4}{|c|}{ Allele } & \multicolumn{6}{|c|}{ Genotype } & \multirow[t]{3}{*}{ HWE test } \\
\hline & \multicolumn{2}{|c|}{ Case } & \multicolumn{2}{|c|}{ Control } & \multicolumn{3}{|c|}{ Case } & \multicolumn{3}{|c|}{ Control } & \\
\hline & $\mathrm{C}$ & $\mathrm{T}$ & $\mathrm{C}$ & $\mathrm{T}$ & $\mathrm{CC}$ & CT & TT & $\mathrm{CC}$ & $\mathrm{CT}$ & TT & \\
\hline Baiqiu et al. (2000) & 35 & 43 & 95 & 63 & 8 & 19 & 12 & 26 & 43 & 10 & $\mathrm{Y}$ \\
\hline Jie et al. (2007) & 111 & 135 & 115 & 143 & 26 & 59 & 38 & 21 & 73 & 35 & $\mathrm{Y}$ \\
\hline Weger et al. (2008) & 396 & 224 & 328 & 166 & 133 & 130 & 47 & 110 & 108 & 29 & $\mathrm{Y}$ \\
\hline Vasku et al. (2009) & 565 & 255 & 305 & 183 & 195 & 175 & 40 & 90 & 125 & 29 & $\mathrm{Y}$ \\
\hline Dehui et al. (2012) & 154 & 62 & 131 & 73 & 60 & 34 & 14 & 42 & 47 & 13 & $\mathrm{Y}$ \\
\hline Liew et al. (2012) & 359 & 41 & 290 & 44 & 159 & 41 & 0 & 125 & 40 & 2 & $\mathrm{Y}$ \\
\hline Asefi et al. (2014) & 143 & 57 & 163 & 37 & 50 & 43 & 7 & 64 & 35 & 1 & $\mathrm{Y}$ \\
\hline
\end{tabular}

$\mathrm{Y}=$ consistent with Hardy-Weinberg equilibrium (HWE). 


\section{Meta-analysis results}

Significant heterogeneity between studies was observed in the comparisons of $\mathrm{C} v s \mathrm{~T}$, $\mathrm{CC} v s \mathrm{CT}+\mathrm{TT}, \mathrm{CC} v s \mathrm{TT}$, and $\mathrm{CC}$ vs CT with the Q-test and the $\mathrm{I}^{2}$ test $\left(\mathrm{P}<0.1\right.$ or $\left.\mathrm{I}^{2}>50 \%\right)$. Therefore, the random effects model was used to pool the results. Meta-analysis results identified that there was no significant difference in MTHFR C677T genotype distribution between psoriasis and control in the comparisons of $\mathrm{C} v s \mathrm{~T}, \mathrm{CC} v s \mathrm{CT}+\mathrm{TT}, \mathrm{CC}+\mathrm{CT} v s \mathrm{TT}, \mathrm{CC} v s \mathrm{TT}$, and $\mathrm{CC} v S \mathrm{CT}$ (respectively: $\mathrm{OR}=0.98,95 \% \mathrm{CI}=0.76-1.26, \mathrm{P}=0.882 ; \mathrm{OR}=1.11,95 \% \mathrm{CI}=$ $0.81-1.51, \mathrm{P}=0.526 ; \mathrm{OR}=0.79,95 \% \mathrm{CI}=0.53-1.19, \mathrm{P}=0.261 ; \mathrm{OR}=0.88,95 \% \mathrm{CI}=0.51-$ $1.52, \mathrm{P}=0.648 ; \mathrm{OR}=1.19,95 \% \mathrm{CI}=0.90-1.58, \mathrm{P}=0.217)($ Table 3$)$.

\begin{tabular}{|c|c|c|c|c|c|c|}
\hline \multirow[t]{2}{*}{ Comparison } & \multirow[t]{2}{*}{ OR } & \multirow[t]{2}{*}{$95 \% \mathrm{CI}$} & \multirow[t]{2}{*}{ P value } & \multicolumn{2}{|c|}{ Heterogeneity } & \multirow[t]{2}{*}{ Effects model } \\
\hline & & & & $\mathrm{I}^{2}$ & $\overline{P \text { value }}$ & \\
\hline $\mathrm{C} v s \mathrm{~T}$ & 0.98 & $0.76-1.26$ & 0.882 & $70.5 \%$ & 0.002 & Random \\
\hline Asian & 0.92 & $0.63-1.33$ & 0.644 & $71.7 \%$ & 0.007 & Random \\
\hline Caucasian & 1.09 & $0.74-1.61$ & 0.654 & $80.6 \%$ & 0.023 & Random \\
\hline $\mathrm{CC} v s \mathrm{CT}+\mathrm{TT}$ & 1.11 & $0.81-1.51$ & 0.526 & $64.1 \%$ & 0.010 & Random \\
\hline Asian & 1.04 & $1.65-1.66$ & 0.881 & $66.3 \%$ & 0.018 & Random \\
\hline Caucasian & 1.21 & $0.74-1.98$ & 0.455 & $77.7 \%$ & 0.034 & Random \\
\hline $\mathrm{CC}+\mathrm{CT} v s \mathrm{TT}$ & 0.79 & $0.53-1.19$ & 0.261 & $45.7 \%$ & 0.087 & Random \\
\hline Asian & 0.65 & $0.33-1.27$ & 0.211 & $48.8 \%$ & 0.099 & Random \\
\hline Caucasian & 0.95 & $0.67-1.36$ & 0.794 & $50.9 \%$ & 0.154 & Random \\
\hline $\mathrm{CC} v s \mathrm{TT}$ & 0.88 & $0.51-1.52$ & 0.648 & $60.8 \%$ & 0.018 & Random \\
\hline Asian & 0.72 & $0.28-1.81$ & 0.480 & $63.7 \%$ & 0.026 & Random \\
\hline Caucasian & 1.08 & $0.52-2.24$ & 0.836 & $73.3 \%$ & 0.053 & Random \\
\hline $\mathrm{CC} v s \mathrm{CT}$ & 1.19 & $0.90-1.58$ & 0.217 & $50.7 \%$ & 0.058 & Random \\
\hline Asian & 1.15 & $0.75-1.75$ & 0.532 & $55.8 \%$ & 0.060 & Random \\
\hline Caucasian & 1.25 & $0.82-1.91$ & 0.298 & $66.0 \%$ & 0.086 & Random \\
\hline
\end{tabular}

$\mathrm{OR}=$ odds ratio; $\mathrm{CI}=$ confidence interval.

In the subgroup analysis based on ethnicity, the studies included were divided into Caucasian and Asian populations, and the results also showed no significant association between MTHFR 677C/T polymorphism and psoriasis risk in both Caucasian and Asian populations. A forest plot of psoriasis risk associated with MTHFR 677C/T polymorphism (CC vs $\mathrm{CT}+\mathrm{TT}$ ) is shown in Figure 2. The results for this and other genetic models are summarized in Table 3.

\section{Sensitivity analysis and publication bias}

Sensitivity analysis was performed by sequential omission of individual studies for all subjects and subgroups. The pooled ORs were not significantly altered in all subjects and subgroups by omitting any single study (data not shown). The results of sensitivity analysis indicated the stability of our results. Begg's funnel plots and the Egger tests were used to assess publication bias. The shape of the funnel plots in the recessive model seemed to be symmetrical (Figure 3), as with the other models (data not shown). The Egger tests also showed that there was no statistically significant publication bias in any genetic model (all $\mathrm{P}>0.05$ ) (data not shown). 


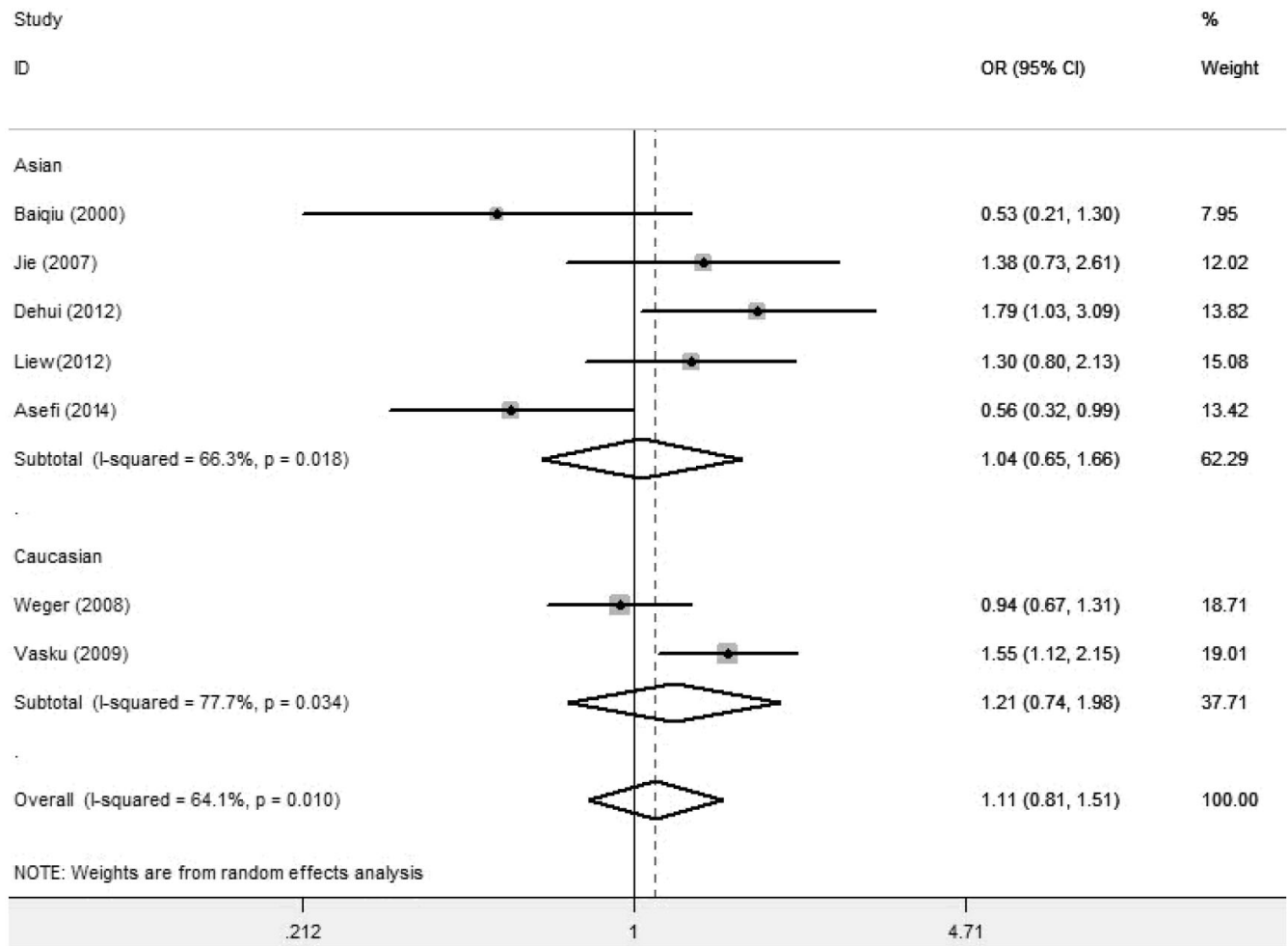

Figure 2. Forest plot of psoriasis risk associated with MTHFR $677 \mathrm{C} / \mathrm{T}$ polymorphism $(\mathrm{CC} v s \mathrm{CT}+\mathrm{TT}) . \mathrm{OR}=$ odds ratio; $\mathrm{CI}=$ confidence interval.

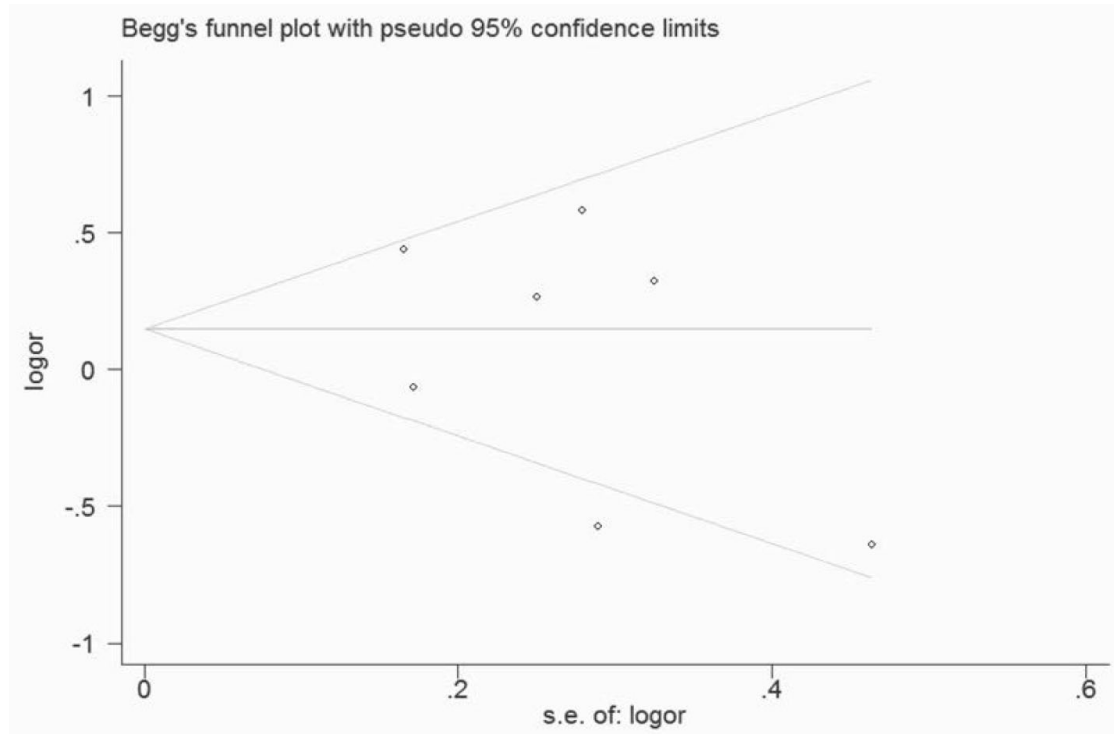

Figure 3. Evaluation of publication bias (CC vs $\mathrm{CT}+\mathrm{TT})$. 


\section{DISCUSSION}

In the last two decades, the association between MTHFR gene polymorphism and psoriasis has attracted great interest amongst researchers worldwide. To date, a number of casecontrol studies have been performed to investigate the association between MTHFR $677 \mathrm{C} / \mathrm{T}$ polymorphism and psoriasis risk (Baiqiu et al., 2000; Jie et al., 2007; Weger et al., 2008; Vasku et al., 2009; Dehui et al., 2012; Liew et al., 2012; Asefi et al., 2014). However, the conclusion was controversial, which may have been partly due to a small sample size in individual studies or differences in various ethnic groups. Meta-analysis has been recognized as a useful statistical method that combines findings from independent studies to precisely evaluate the effect of selected genetic polymorphism on the risk of disease (Attia et al., 2003). To the best of our knowledge, no meta-analysis has been conducted to evaluate the association between MTHFR C677T polymorphism and psoriasis risk. Therefore, there is a need to perform a meta-analysis using published data to clarify inconsistent findings.

Based on seven case-control studies involving 1290 psoriasis cases and 1068 healthy controls, this meta-analysis revealed that there was no significant difference in MTHFR C677T genotype distribution between psoriasis and control subjects in the comparisons of $\mathrm{C} v s \mathrm{~T}, \mathrm{CC}$ vs $\mathrm{CT}+\mathrm{TT}, \mathrm{CC}+\mathrm{CT}$ vs TT, $\mathrm{CC}$ vs $\mathrm{TT}$, and $\mathrm{CC}$ vs $\mathrm{CT}$, which indicated that there may be no association between MTHFR 677C/T polymorphism and psoriasis risk. Similarly, in the subgroup analysis by ethnicity, no significant association was found between MTHFR C677T polymorphism and psoriasis risk in all comparisons. Results showed obvious heterogeneity between studies, suggesting a possible role of ethnic difference in genetic backgrounds and the environment in which the subjects lived. As the eligible study number was small in this meta-analysis of MTHFR $677 \mathrm{C} / \mathrm{T}$ polymorphism, these results still need further investigation.

Some limitations of our meta-analysis should be acknowledged. First, because of incomplete raw data on publication, some relevant studies could not be included in our analysis. Second, the number of included studies was not sufficiently large and the sample sizes of some of the included studies were relatively small, which may not provide enough statistical power to explore the real association between MTHFR $677 \mathrm{C} / \mathrm{T}$ polymorphism and psoriasis risk. Third, the included publications were limited to Asian and Caucasian populations, so future work should examine other populations. Fourth, although no obvious publication bias was identified, potential bias cannot be completely ruled out. Finally, our results were based on unadjusted data and lacked the information for the data analysis, which may have caused serious confounding bias.

In summary, our meta-analysis of seven case-control studies suggested that MTHFR $677 \mathrm{C} / \mathrm{T}$ polymorphism may not be associated with psoriasis risk. As few studies are available in this field and current evidence remains limited, further studies are still needed to warrant and validate our results.

\section{REFERENCES}

Asefi M, Vaisi-Raygani A, Khodarahmi R, Nemati H, et al. (2014). Methylentetrahydrofolatereductase (rs1801133) polymorphism and psoriasis: contribution to oxidative stress, lipid peroxidation and correlation with vascular adhesion protein 1, preliminary report. J. Eur. Acad. Dermatol. Venereol. 28: 1192-1198.

Attia J, Thakkinstian A and D'Este C (2003). Meta-analyses of molecular association studies: methodologic lessons for genetic epidemiology. J. Clin. Epidemiol. 56: 297-303. 
Baiqiu W, Songbin F, Guiyin Z and Pu L (2000). Study of the relationship between psoriasis and the polymorphic site C677T of methylenetetrahydrofolate reductase. Chin. Med. Sci. J. 15: 119-120.

Dehui J, Yali S and Zhanxiang W (2012). The methylenetetrahydrofolate reductase $677 \mathrm{C}>\mathrm{T}$ gene polymorphism in psoriatic patients and relationship with efficacy and toxicity of methotrexate. China J. Leprosy Skin Diseases 28: 459-461.

Friso S, Choi SW, Girelli D, Mason JB, et al. (2002). A common mutation in the 5,10-methylenetetrahydrofolate reductase gene affects genomic DNA methylation through an interaction with folate status. Proc. Natl. Acad. Sci. U. S. A. 99: 5606-5611.

Higgins JP and Thompson SG (2002). Quantifying heterogeneity in a meta-analysis. Stat. Med. 21: 1539-1558.

Jie W, Yuzhen S, Hongyu M, Jing B, et al. (2007). Association between single nucleotide polymorphisms of MTHFR gene and susceptibility of psoriasis. Int. J. Genet. 30: 173-177.

Krueger JG and Bowcock A (2005). Psoriasis pathophysiology: current concepts of pathogenesis. Ann. Rheum. Dis. 64: ii30-ii36.

Krueger JG, Krane JF, Carter DM and Gottlieb AB (1990). Role of growth factors, cytokines, and their receptors in the pathogenesis of psoriasis. J. Invest. Dermatol. 94: 135S-140S.

Liew SC, Das-Gupta E, Wong SF, Lee N, et al. (2012). Association of methylentetraydrofolate reductase (MTHFR) 677 C $>$ T gene polymorphism and homocysteine levels in psoriasis vulgaris patients from Malaysia: a case-control study. Nutr. J. 11:1.

Ni C and Chiu MW (2014). Psoriasis and comorbidities: links and risks. Clin. Cosmet. Investig. Dermatol. 7: 119-132.

Peters JL, Sutton AJ, Jones DR, Abrams KR, et al. (2006). Comparison of two methods to detect publication bias in metaanalysis. JAMA 295: 676-680.

Ruchusatsawat K, Wongpiyabovorn J, Shuangshoti S, Hirankarn N, et al. (2006). SHP-1 promoter 2 methylation in normal epithelial tissues and demethylation in psoriasis. J. Mol. Med. 84: 175-182.

Schaid DJ and Jacobsen SJ (1999). Biased tests of association: comparisons of allele frequencies when departing from Hardy-Weinberg proportions. Am. J. Epidemiol. 149: 706-711.

Selhub J and Miller JW (1992). The pathogenesis of homocysteinemia: interruption of the coordinate regulation by S-adenosylmethionine of the remethylation and transsulfuration of homocysteine. Am. J. Clin. Nutr. 55: 131-138.

Stern LL, Mason JB, Selhub J and Choi SW (2000). Genomic DNA hypomethylation, a characteristic of most cancers, is present in peripheral leukocytes of individuals who are homozygous for the C677T polymorphism in the methylenetetrahydrofolate reductase gene. Cancer Epidemiol. Biomarkers Prev. 9: 849-853.

Vasku V, Bienertova-Vasku J, Necas M and Vasku A (2009). MTHFR (methylenetetrahydrofolate reductase) C677T polymorphism and psoriasis. Clin. Exp. Med. 9: 327-331.

Weger W, Hofer A, Stanger O, Wolf P, et al. (2008). The methylenetetrahydrofolate reductase $677 \mathrm{C}>\mathrm{T}$ gene polymorphism is not associated with chronic plaque psoriasis. Exp. Dermatol. 17: 748-751.

Zenz R, Eferl R, Kenner L, Florin L, et al. (2005). Psoriasis-like skin disease and arthritis caused by inducible epidermal deletion of Jun proteins. Nature 437: 369-375.

Zhang K, Zhang R, Li X, Yin G, et al. (2007). The mRNA expression and promoter methylation status of the p16 gene in colony-forming cells with high proliferative potential in patients with psoriasis. Clin. Exp. Dermatol. 32: 702-708.

Zintzaras E and Ioannidis JP (2005). Heterogeneity testing in meta-analysis of genome searches. Genet. Epidemiol. 28: 123-137. 